Virginia Commonwealth University VCU Scholars Compass

2011

\title{
Intrinsic ferromagnetism in two-dimensional carbon structures: Triangular graphene nanoflakes linked by carbon chains
}

Jian Zhou

Peking University

Qian Wang

Peking University

Qiang Sun

Peking University

Puru Jena

Virginia Commonwealth University, pjena@vcu.edu

Follow this and additional works at: http://scholarscompass.vcu.edu/phys_pubs

Zhou, J., Wang, Q. Sun, Q. et al. Intrinsic ferromagnetism in two-dimensional carbon structures: Triangular graphene nanoflakes linked by carbon chains. Physical Review B, 84, 081402(R) (2011). Copyright (C) 2011 American Physical Society.

\section{Downloaded from}

http://scholarscompass.vcu.edu/phys_pubs/61

This Article is brought to you for free and open access by the Dept. of Physics at VCU Scholars Compass. It has been accepted for inclusion in Physics Publications by an authorized administrator of VCU Scholars Compass. For more information, please contact libcompass@vcu.edu. 


\title{
Intrinsic ferromagnetism in two-dimensional carbon structures: Triangular graphene nanoflakes linked by carbon chains
}

\author{
Jian Zhou, ${ }^{1,2}$ Qian Wang, ${ }^{2,3}$ Qiang Sun,,${ }^{1,2,3, *}$ and Puru Jena ${ }^{3}$ \\ ${ }^{1}$ Department of Advanced Materials and Nanotechnology, College of Engineering, Peking University, Beijing 100871, China \\ ${ }^{2}$ Center for Applied Physics and Technology, Peking University, Beijing 100871, China \\ ${ }^{3}$ Department of Physics, Virginia Commonwealth University, Richmond, Virginia 23284, USA
}

(Received 24 June 2011; published 5 August 2011)

\begin{abstract}
Using density functional theory (DFT) we show that intrinsic ferromagnetism in two-dimensional (2D) carbon semiconducting structures can be achieved by linking triangular graphene nanoflakes (GNFs) with carbon chains containing an odd number of carbon atoms. The observed magnetism can be understood from the singlet-triplet rule of $\mathrm{C}$ chain, the anti-pattern rule for a magnetic bipartite $\mathrm{C}$ structure, and the Lieb-Mattis criterion. Monte Carlo (MC) simulations indicate that the 2D frameworks can display transitions from a high-spin state to a low-spin state and to a paramagnetic state as temperature increases.
\end{abstract}

DOI: 10.1103/PhysRevB.84.081402

PACS number(s): 81.05.ue, 73.22.Pr, 75.75.-c

Graphene sheet ${ }^{1}$ and its related structures show many unique properties; among them magnetism is very interesting. ${ }^{2}$ It has been confirmed that zigzag-edge states of graphene nanoribbon (GNR) contribute to magnetism. ${ }^{3}$ In fact there are two important magnetic structures related to zigzag-edge states: GNR and a triangular graphene nanoflake (GNF), both of which can be experimentally obtained by various cutting techniques. ${ }^{4,5}$ However, these two structures display different magnetic behavior: GNR structures show antiferromagnetic (AFM) coupling between the two edges. Although GNFs exhibit ferromagnetic (FM) coupling among three zigzag edges, ${ }^{5}$ the coupling between GNFs becomes AFM when they are directly assembled. ${ }^{6,7}$ Recent experiments showed that graphene can exhibit intrinsic ferromagnetism. ${ }^{8}$ On the other hand Sepioni et al. found no FM state in their graphene samples, but only weak paramagnetism (PM) was observed. ${ }^{9}$ The questions here are: How does one understand these conflicting experimental results? Can two-dimensional (2D) graphene-based structures show ferromagnetism without addition of any other impurities?

In this Rapid Communication, we show that 2D FM (PM) carbon structures can be achieved by linking GNFs with odd(even-)numbered carbon chains. Such structures can be realized in experiments using electron-beam irradiation, etching techniques, ${ }^{10}$ or block copolymer lithography ${ }^{11}$ techniques.

Our calculations are based on DFT with the Perdew-BurkeErnzerhost (PBE) $)^{12}$ form for the generalized gradient approximation for exchange correlation functional, as implemented in the Vienna ab initio simulation package. ${ }^{13,14}$ We use a vacuum space of $12 \AA$ to avoid interactions between two images. $\Gamma$ point and $5 \times 5 \times 1$ Monkhorst-Pack special-k-point meshes ${ }^{15}$ are applied to represent the reciprocal space for OD cluster and 2D periodic framework structures, respectively. Convergence of total energy, Hellman-Feynman force, and energy cutoff are set to be $1 \times 10^{-4} \mathrm{eV}, 0.01 \mathrm{eV} / \AA$, and $400 \mathrm{eV}$, respectively. The accuracy of our theoretical procedure has been tested in a previous paper. ${ }^{16}$

First we examine the electronic and magnetic properties of isolated triangular GNFs. According to Lieb's theorem, ${ }^{17}$ GNF with $m$ zigzag C-atom lines $\left(\mathrm{GNF}_{m}\right)$ always exhibits $(m-2)$ magnetic moments. For example, our results show that the total magnetic moments of $\mathrm{GNF}_{3}$ and $\mathrm{GNF}_{5}$ are, respectively, $1 \mu_{B}$ and $3 \mu_{B}$, both consistent with Lieb's theorem.

Next we consider the magnetic coupling between two GNFs. Here $\mathrm{GNF}_{5}$ and $\mathrm{GNF}_{3}$ are selected as test cases. As shown in Fig. 1(a) and (b), when two $G F_{5}$ s are linked directly with each other, they prefer AFM coupling $\left(0 \mu_{B}\right)$, which is $47 \mathrm{meV}$ lower in energy than that with FM coupling. In contrast, if one $\mathrm{C}$ atom is introduced between them, the FM state becomes more favorable than the AFM state by $196 \mathrm{meV}$, and the total magnetic moment becomes $8 \mu_{B}$. The corresponding results of $\mathrm{GNF}_{3}$ are shown in Fig. 1(c) and (d), where the ground state for $\mathrm{GNF}_{3}-\mathrm{GNF}_{3}$ and $\mathrm{GNF}_{3}-\mathrm{C}_{1}-\mathrm{GNF}_{3}$ has $0 \mu_{\mathrm{B}}$ (AFM is $38 \mathrm{meV}$ lower than FM) and $4 \mu_{B}$ (FM is $165 \mathrm{meV}$ lower than AFM), respectively. These findings suggest that the magnetic coupling between GNFs can be mediated by introducing $\mathrm{C}$ atoms. The next question is what would happen if we increase the number of $\mathrm{C}$ atoms forming a $\mathrm{C}$ chain? To answer this we recall the "singlet-triplet rule" found in $\mathrm{C}$ chains. ${ }^{18}$ This rule states that a single hydrogenterminated carbon chain with an odd (even) number of $\mathrm{C}$ atoms has the triplet (singlet) ground state. Based on these findings we can expect that the magnetic coupling between GNFs can be modulated by changing the chain length.

Geometric structure calculations of $\mathrm{GNF}_{5}-\mathrm{C}_{n}-\mathrm{GNF}_{5}$ show that the distributions of bond lengths of $\mathrm{C}-\mathrm{C}$ on $\mathrm{C}$ chain are similar to that in an isolated hydrogenated $\mathrm{C}$ chain. ${ }^{19}$ This indicates that the $\mathrm{C}$ chain between the two GNFs behaves more similarly to a $\mathrm{H}$-terminated polyyine than to a $\mathrm{H}_{2}$-terminated chain. ${ }^{20}$ We now focus on the magnetic coupling between two $\mathrm{GNF}_{5} \mathrm{~s}$. It is found that the magnetic couplings are different depending on $n$. We plot exchange energy $\left(E_{\mathrm{AFM}}-E_{\mathrm{FM}}\right)$ for $n=0$ to 7 in Fig. 2(a). As can be seen odd (even) $n$ leads to FM (AFM) coupling between two $\mathrm{GNF}_{5}$ s. The spin density of $\mathrm{GNF}_{5}-\mathrm{C}_{5}-\mathrm{GNF}_{5}$ in FM state and $\mathrm{GNF}_{5}-\mathrm{C}_{4}-\mathrm{GNF}_{5}$ in AFM state are plotted in Fig. 2(b). For odd $n$, each $\mathrm{GNF}_{5}$ contributes $3 \mu_{B}$, and the $\mathrm{C}$ chain contributes $2 \mu_{B}$. Thus, the total magnetic moment of it is $(3+2+3=) 8 \mu_{B}$ [Fig. 2(c)]. On the other hand for even $n=0$ the system shows stable AFM-singlet ground state with exchange energy of $-47 \mathrm{meV}$. For $n=2,4$, and 6 , the exchange 


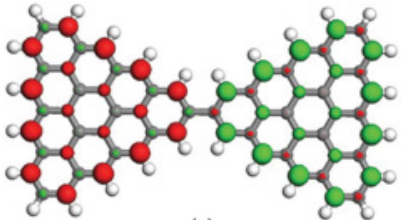

(a)

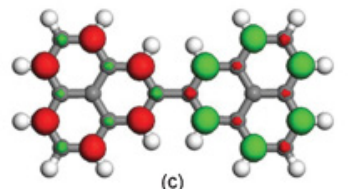

(b)

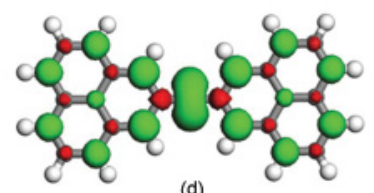

(d)

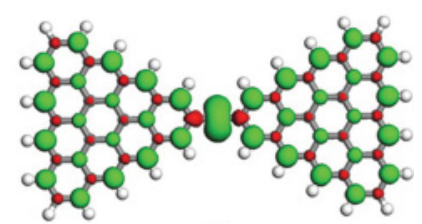

FIG. 1. (Color online) Geometric structures of (a) $\mathrm{GNF}_{5}-\mathrm{GNF}_{5}$, (b) $\mathrm{GNF}_{5}-\mathrm{C}_{1}-\mathrm{GNF}_{5}$, (c) $\mathrm{GNF}_{3}-\mathrm{GNF}_{3}$, and (d) $\mathrm{GNF}_{3}-\mathrm{C}_{1}-\mathrm{GNF}_{3}$ along with their spin density $\left(\rho_{\alpha}-\rho_{\beta}\right)$ isosurface with value of 0.03 electron $/ \AA^{3}$ in their own stable spin configurations, AFM and $\mathrm{FM}$, respectively. Green and red represent positive and negative signs, respectively.

energy is negative and very small (absolute value smaller than $10 \mathrm{meV}$ ) and approaches zero for larger $n$, indicating nearly degenerate FM and AFM states. In these even $n$ cases the $\mathrm{C}$ chains are nearly NM [Fig. 2(c)], similar to that seen in hydrogen-terminated finite $\mathrm{C}$ chains $\mathrm{C}_{2 m} \mathrm{H}_{2} \cdot{ }^{18}$ Hence, the total magnetic moment of $\mathrm{GNF}_{5}-\mathrm{C}_{n}-\mathrm{GNF}_{5}$ is $0 \mu_{B}(3+0-3)$ when $n$ is even.

For a better understanding of our results, it is helpful to note the following pattern rule (termed as an antipattern rule): magnetic $\mathrm{C}$ atom favors to couple ferrimagnetically [ $\uparrow \downarrow]$ with its nearest neighbor magnetic $\mathrm{C}$ or antiferromagnetically in bipartite $\mathrm{C}$-based nanomaterials including zigzag edged GNR, ${ }^{21}$ partially hydrogenated graphene sheet, ${ }^{22,23}$ GNF,, 7 etc. To obey the rule two GNFs should have parallel spin direction when linked by a triplet chain with an odd number of $\mathrm{C}$ atoms, showing a coupling configuration like $[\uparrow \downarrow \uparrow \downarrow \uparrow]$. Here, the central three arrows represent the $\mathrm{C}$ chain. We observe that the $2-\mu_{\mathrm{B}}$ magnetic moment is distributed almost uniformly on the $\mathrm{C}$ chain. The two end $\mathrm{C}$ atoms of the chain carry less and less magnetic moment as $n$ gets larger, so the coupling strength between the $\mathrm{GNF}_{5}$ and the $\mathrm{C}$ chain decreases monotonically. This leads to a decrease of the exchange energy. On the other hand even-numbered isolated $\mathrm{C}$ chains have NM-singlet ground states, and when inserted between two GNFs they are only slightly magnetically polarized by $\mathrm{GNF}_{5}$. Thus, the ability to mediate magnetic coupling is weak. The magnetic coupling configurations $[\uparrow \ldots \downarrow]$ and $[\uparrow \ldots \uparrow]$ are nearly degenerate for $n=2,4,6$. But for $n=0$, these two $\mathrm{GNF}_{5} \mathrm{~s}$ are directly linked. The antipattern rule requires AFM coupling between two $\mathrm{GNF}_{5} \mathrm{~s}$. We should emphasize that the above rule may not be valid for $\mathrm{C}$ chain coupled with other foreign elements, e.g., d-orbital transition-metal atoms, ${ }^{24}$ since some other magnetic coupling mechanisms may exist between foreign atoms.

The total magnetic moment of the systems cannot be derived from the Hubbard model based on Lieb's theorem ${ }^{17}$ where the Coulomb energy $U$ should be positive and constant

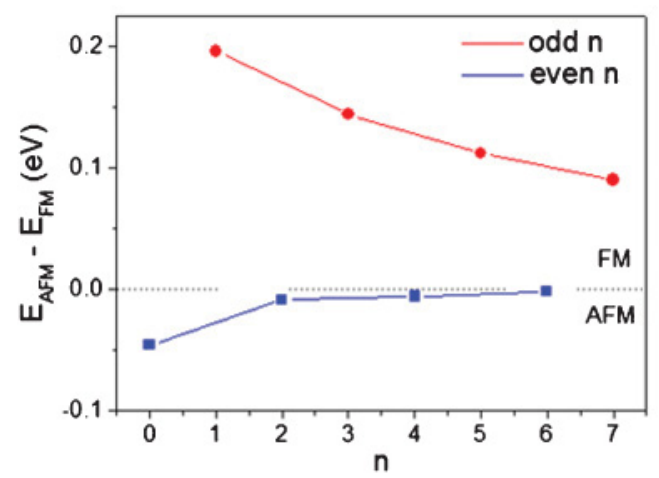

(a)

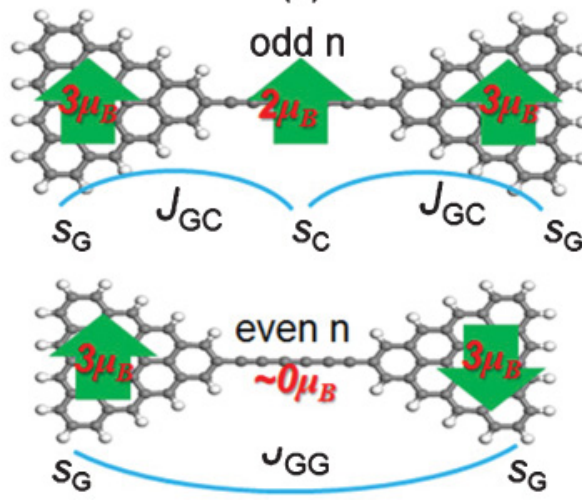

(c)
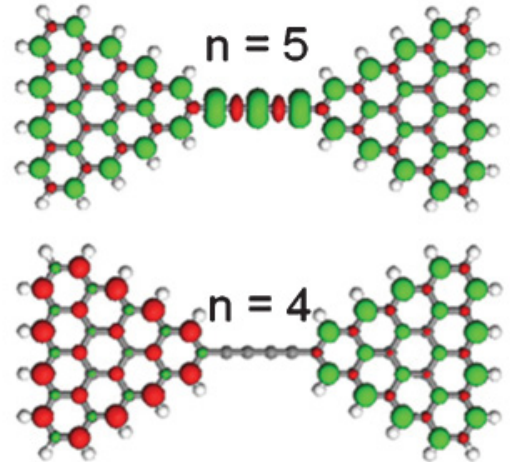

(b)

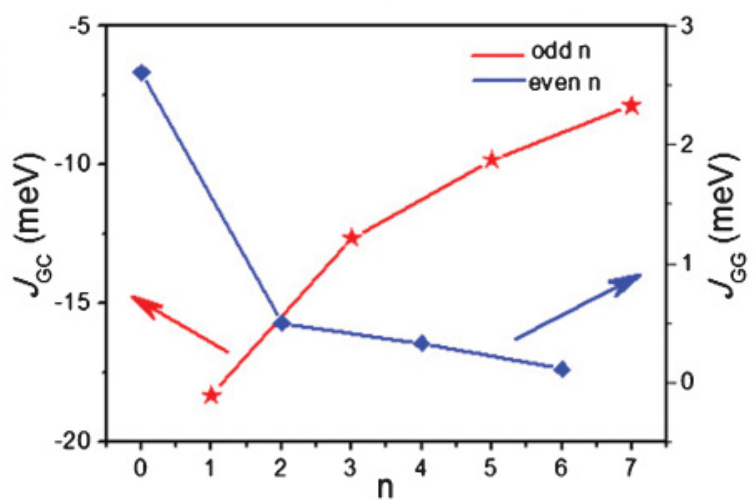

(d)

FIG. 2. (Color online) (a) Exchange energy $\left(E_{\mathrm{AFM}}-E_{\mathrm{FM}}\right)$ as a function of $\mathrm{C}$ chain index $n$. (b) Spin density of $\mathrm{GNF}_{5}-\mathrm{C}_{5}-\mathrm{GNF}_{5}$ in $\mathrm{FM}$ and $\mathrm{GNF}_{5}-\mathrm{C}_{4}-\mathrm{GNF}_{5}$ in AFM states. (c) Sketch of Ising model describing magnetic behavior with exchange parameters for odd and even $n$. (d) Simulated variation of $J_{\mathrm{GC}}$ and $J_{\mathrm{GG}}$ parameters with index $n$. 
with respect to all sites and each site can only have one unpaired electron. On the other hand we notice that the present antipattern systems satisfy the Lieb-Mattis condition of $g=$ $0,{ }^{25}$ where the authors applied a Heisenberg magnetic model. In their work the total spin of the systems can be derived by $\mathcal{F}=\left|S_{\mathrm{A}}-S_{\mathrm{B}}\right|$, where $S_{\mathrm{A}(\mathrm{B})}$ represents the maximum possible spin of subset A (B). Therefore, we can evaluate $S_{\mathrm{A}(\mathrm{B})}$ and $\mathcal{S}$ in our systems. Taking $\mathrm{GNF}_{5}-\mathrm{C}_{5}-\mathrm{GNF}_{5}$ as example, the maximum possible spins of each $\mathrm{C}$ atom on the GNF and the chain are $1 \mu_{B}$ and $2 \mu_{B}$, respectively, depending on their coordination numbers. Thus, $S_{\mathrm{A}}=18 \times 1+3 \times 2+18$ $\times 1=42 \mu_{B}$ and $S_{\mathrm{B}}=15 \times 1+2 \times 2+15 \times 1=34 \mu_{B}$. Hence, $\stackrel{\mathcal{S}}{=}=\left|S_{\mathrm{A}}-S_{\mathrm{B}}\right|=8 \mu_{B}$, which is consistent with our first principle result. For $\mathrm{GNF}_{5}-\mathrm{C}_{4}-\mathrm{GNF}_{5}$, similar procedure can be applied to demonstrate the AFM coupling of the ground state $\left(S_{\mathrm{A}}=18 \times 1+2 \times 2+15 \times 1=37 \mu_{B}\right.$ and $S_{\mathrm{B}}=$ $15 \times 1+2 \times 2+18 \times 1=37 \mu_{B}$, thus $\mathcal{F}=\left|S_{\mathrm{A}}-S_{\mathrm{B}}\right|=$ $\left.0 \mu_{B}\right)$.

In order to understand the magnetic coupling between the GNF and the $\mathrm{C}$ chain quantitatively we use the Ising model to calculate the exchange parameters $J_{\mathrm{GC}}$ and $J_{\mathrm{GG}}$ [Fig. 2(c)]. Symbols $s_{\mathrm{G}}$ and $s_{\mathrm{C}}$ denote the total magnetic moment of GNF and $\mathrm{C}$ chain, respectively. In the Ising model the Hamiltonian can be written as $H=H_{0}+\sum_{\langle i, j\rangle} J_{i j} s_{i} s_{j}$, where $J_{i j}$ is the exchange parameter between $i$ and $j . J_{\mathrm{GC}}$ and $J_{\mathrm{GG}}$ can be determined through the following energy calculations. For systems with an odd-numbered $\mathrm{C}$ chain, we use two different magnetic configurations: $\left(s_{\mathrm{G}}, s_{\mathrm{C}}, s_{\mathrm{G}}\right)=(3,2,3)$ and $(3,-2,3)$, with corresponding total energy denoted as $E_{1}$ and $E_{2}$, respectively. Therefore, $J_{\mathrm{GC}}$ can be obtained through $J_{\mathrm{GC}}=$ $\left(E_{1}-E_{2} /\right) 4 s_{\mathrm{G}} s_{\mathrm{C}}$. For even $n$ the exchange parameter $J_{\mathrm{GG}}=$ $\left(E_{\mathrm{FM}}-E_{\mathrm{AFM}} /\right) 2 s_{\mathrm{G}} s_{\mathrm{G}}$. As plotted in Fig. 2(d), $J_{\mathrm{GC}}$ is negative ranging between $-18 \mathrm{meV}$ and $-8 \mathrm{meV}$. For even $n$ only $n=0$ case has a relatively large exchange parameter $J_{\mathrm{GG}}$ $(=2.6 \mathrm{meV})$, indicating AFM coupling. For $n=2,4,6$, we can see that $J_{\mathrm{GG}}$ is rather small, namely, $<0.5 \mathrm{meV}$. Thus, the systems will exhibit magnetic duality.

Finally, we build a periodic $2 \mathrm{D}$ framework using $\mathrm{GNF}_{3}-$ $\mathrm{C}_{3}-\mathrm{GNF}_{3}$ (Fig. 3). We also studied periodic $\mathrm{GNF}_{3}-\mathrm{C}_{4}-\mathrm{GNF}_{3}$ framework. It is again found that FM and AFM states are nearly degenerate in energy, consistent with the observation of weak PM in Ref. 9. We again find that two $\mathrm{GNF}_{3}$ s carrying $1 \mu_{B}$ each couple in FM state [Fig. 3(b)], which is favored over the AFM state by $0.354 \mathrm{eV} /$ unit. Each $\mathrm{C}_{3}$ chain continues to

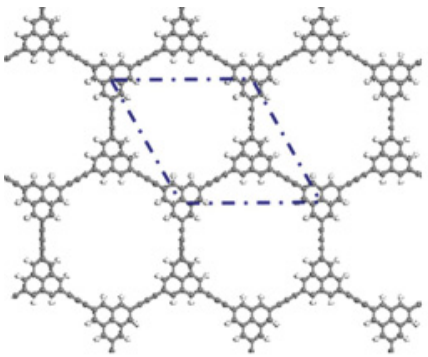

(a)

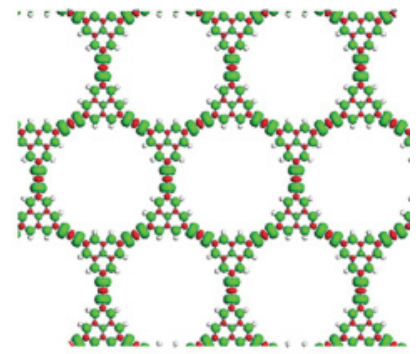

(b)
FIG. 3. (Color online) (a) Geometric structure and (b) spin density isosurface of $2 \mathrm{D}$ periodic $\mathrm{GNF}_{3}-\mathrm{C}_{3}-\mathrm{GNF}_{3}$ framework. Bluedashed rhombus denotes the simulated unit cell. contribute $2 \mu_{B}$ to the magnetic moment. Therefore, in one unit cell we can obtain a total magnetic moment of $(2 \times 1+$ $3 \times 2=) 8 \mu_{B}$. This confirms that the previous magnetic coupling results are also valid for periodic $2 \mathrm{D}$ systems and consistent with experimental observations. ${ }^{8}$ We note that this novel porous graphene-based sheet can show large and stable ferromagnetism. Band-structure calculation shows that the system is semiconducting with a $1.10 \mathrm{eV}$ bandgap. ${ }^{19}$ To check the stability of such periodic systems, we performed molecular dynamics simulations using Nose-Hoover heat bath at $300 \mathrm{~K}$ for $3000 \mathrm{fs}$. After simulation, the system retains its structure with only slight distortions, which can be relaxed back to its original form through geometry optimization. Also, we considered the reaction of periodic $\mathrm{GNF}_{3}-\mathrm{C}_{3}-\mathrm{GNF}_{3}$ with $\mathrm{H}_{2}$ as well as $\mathrm{O}_{2}$ molecules. It is found that $\mathrm{H}_{2}$ interacts weakly with the system and does not affect FM properties, while $\mathrm{O}_{2}$ bonds with the $\mathrm{C}$ chain and quenches its magnetism. ${ }^{19}$ Therefore, oxygen contamination should be avoided.

We also need to point out that the two GNFs linked by a carbon chain can experience relative rotations, and the energy will change with the dihedral angle. However, the nature of magnetic coupling remained unchanged. ${ }^{19}$ But for a periodic $2 \mathrm{D}$ structure the system becomes stiff, and the relative rotation will be restrained.

In order to examine the magnetic behavior of this periodic framework at finite temperatures, we carried out MC simulations for a $(10 \times 10)$ supercell system using the Ising model. $1 \times 10^{5}$ steps are used to analyze the data. Total energy of the system is estimated using the Ising model discussed previously, which can capture the basic magnetic properties of the systems. We plot the variations of total magnetization $\langle M\rangle=\left\langle\sum_{i} s_{i}\right\rangle$ per unit cell with respect to temperature. Multispin states are found with magnetic moment of 8,6 , and $0 \mu_{B}$ as temperature increases [Fig. 4(a)]. The low-spin state of $6 \mu_{B}$ is observed between $180 \sim 280 \mathrm{~K}$, which is attributable to the thermal spin flipping in one carbon chain in the unit cell. When temperature increases further, no net magnetic moment was observed. The Curie temperature is found to be $\sim 440 \mathrm{~K}$. To check whether such multistate spin configuration phenomenon is a common feature for all periodic systems, we performed $\mathrm{MC}$ simulations for $2 \mathrm{D}$ periodic $\mathrm{GNF}_{5}-\mathrm{C}_{7}-\mathrm{GNF}_{5}$ [Fig. 4(b)]. As observed, it also has high-spin state $\left(12 \mu_{B}\right.$ when temperature is below $\sim 80 \mathrm{~K})$, low-spin state $\left(10 \mu_{B}\right.$ when temperature is between $\sim 100$ and $\sim 200 \mathrm{~K}$ ), and paramagnetic state as temperature varies. Beyond $200 \mathrm{~K}$, magnetization gradually decreases as temperature increases. Therefore, we conclude that the multispin-state property is a common feature for 2D periodic framework composed of GNFs and C chains.

In summary we have systematically studied the magnetic property of GNFs linked by $\mathrm{C}$ chains. We found that even and odd numbered $\mathrm{C}$ chains lead to different magnetic coupling between GNFs. Odd-numbered magnetic $\mathrm{C}$ chains result in FM coupling between GNFs, while even-numbered $\mathrm{C}$ chains do not. The underlying mechanisms can be understood from the singlet-triplet rule of $\mathrm{C}$ chain, the anti-pattern rule for a magnetic bipartite $\mathrm{C}$ structure, and the Lieb-Mattis criterion determining the total magnetic moment. $\mathrm{MC}$ simulation further shows that, depending on temperature, the 2D framework can display rich magnetic properties: high-spin, low-spin, 


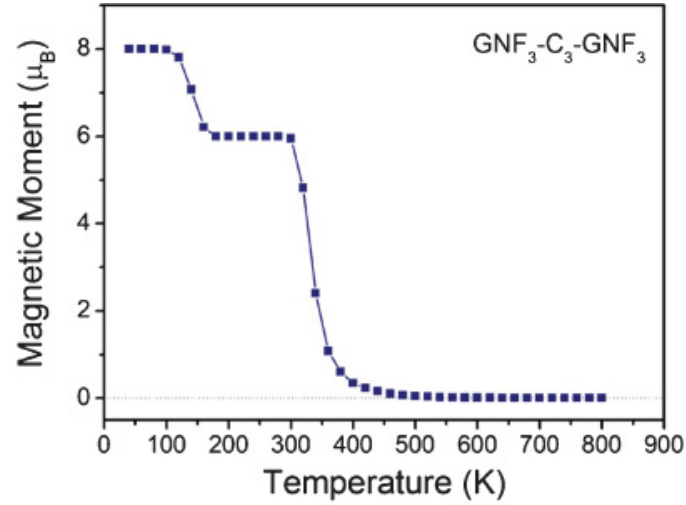

(a)

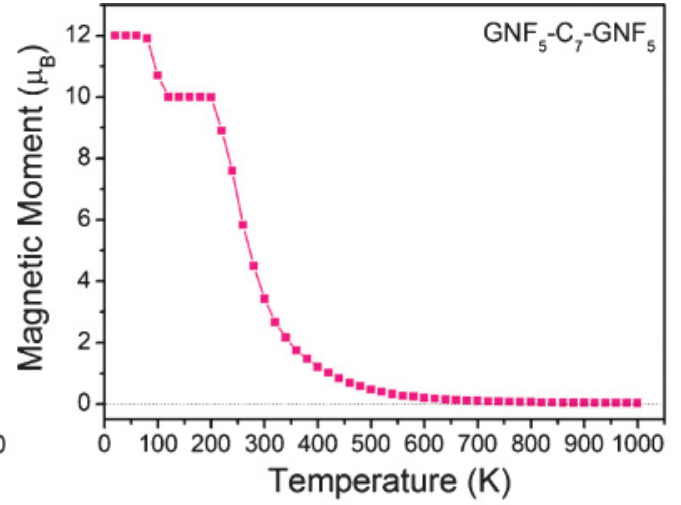

(b)

FIG. 4. (Color online) Variation of magnetization per unit cell of $2 \mathrm{D}$ periodic (a) $\mathrm{GNF}_{3}-\mathrm{C}_{3}-\mathrm{GNF}_{3}$ and (b) $\mathrm{GNF}_{5}-\mathrm{C}_{7}-\mathrm{GNF}_{5}$ as a function of temperature.

and nonspin states. The corresponding Curie temperature is approximately $440 \mathrm{~K}$ for periodic $\mathrm{GNF}_{3}-\mathrm{C}_{3}-\mathrm{GNF}_{3}$ system and can be tuned by changing the size of GNFs and the length of the $\mathrm{C}$ chains.
This work is partially supported by Grants from the National Natural Science Foundation of China (Grants No. NSFC-10744006 and No. NSFC-10874007) and from the US Department of Energy.
*Corresponding author: sunqiang@pku.edu.cn

${ }^{1}$ K. S. Novoselov, A. K. Geim, S. V. Morozov, D. Jiang, Y. Zhang, S. V. Dubonos, I. V. Grigorieva, and A. A. Filrsov, Science 306, 666 (2004).

${ }^{2}$ O. V. Yazyev, Rep. Prog. Phys. 73, 056501 (2010).

${ }^{3}$ V. L. Joseph Joly, M. Kiguchi, S. J. Hao, K. Takai, T. Enoki, R. Sumii, K. Amemiya, H. Muramatsu, T. Hayashi, Y. A. Kim, M. Endo, J. Campos-Delgado, F. Lopez-Urias, A. Botello-Mendez, H. Terrones, M. Terrones, and M. S. Dresselhaus, Phys. Rev. B 81, 245428 (2010).

${ }^{4}$ L. Jiao, L. Zhang, X. Wang, G. Diankov, and H. Dai, Nature 458, 877 (2009).

${ }^{5}$ J. Inoue, K. Fukui, T. Kubo, S. Nakazawa, K. Sato, D. Shiomi, Y. Morita, K. Yamamoto, T. Takui, and K. Nakasuji, J. Am. Chem. Soc. 123, 12702 (2001).

${ }^{6}$ O. V. Yazyev, W. L. Wang, S. Meng, and E. Kaxiras, Nano Lett. 8, 766 (2008).

${ }^{7}$ W. L. Wang, O. V. Yazyev, S. Meng, and E. Kaxiras, Phys. Rev. Lett. 102, 157201 (2009).

${ }^{8}$ Y. Wang, Y. Huang, Y. Song, X. Zhang, Y. Ma, J. Liang, and Y. Chen, Nano Lett. 9, 220 (2009).

${ }^{9}$ M. Sepioni, R. R. Nair, S. Rablen, J. Narayanan, F. Tuna, R. Winpenny, A. K. Geim, and I. V. Grigorieva, Phys. Rev. Lett. 105, 207205 (2010).

${ }^{10}$ C. Jin, H. Lan, L. Peng, K. Suenage, and S. Iijima, Phys. Rev. Lett. 102, 205501 (2009).
${ }^{11}$ J. Bai, X. Zhong, S. Jiang, Y. Huang, and X. Duan, Nat. Nanotechnol. 5, 190 (2010).

${ }^{12}$ J. P. Perdew, K. Burke, and M. Ernzerhof, Phys. Rev. Lett. 77, 3865 (1996).

${ }^{13}$ G. Kresse and J. Furthmuller, Phys. Rev. B 54, 11169 (1996).

${ }^{14}$ G. Kresse and D. Joubert, Phys. Rev. B 59, 1758 (1999).

${ }^{15}$ H. J. Monkhorst and J. D. Pack, Phys. Rev. B 13, 5188 (1976).

${ }^{16}$ J. Zhou, Q. Wang, Q. Sun, X. S. Chen, Y. Kawazoe, and P. Jena, Nano Lett. 9, 3867 (2009).

${ }^{17}$ E. H. Lieb, Phys. Rev. Lett. 62, 1201 (1989).

${ }^{18}$ X. Fan, L. Liu, J. Lin, Z. Shen, and J.-L. Kuo, ACS Nano 3, 3788 (2009).

${ }^{19}$ See Supplemental Material at http://link.aps.org/supplemental/ 10.1103/PhysRevB.84.081402 for geometric, electronic structure, adsorption, rotation, and formation energy properties of the systems.

${ }^{20}$ L. Ravagnan, N. Manini, E. Cinquanta, G. Onida, D. Sangalli, C. Motta, M. Devetta, A. Bordoni, P. Piseri, and P. Milani, Phys. Rev. Lett. 102, 245502 (2009).

${ }^{21}$ Y.-W. Son, M. L. Cohen, and S. G. Louie, Phys. Rev. Lett. 97, 216803 (2006).

${ }^{22}$ J. Zhou, M. M. Wu, X. Zhou, and Q. Sun, Appl. Phys. Lett. 95, 103108 (2009).

${ }^{23}$ O. V. Yazyev and L. Helm, Phys. Rev. B 75, 125408 (2007).

${ }^{24}$ E. Durgun, R. T. Senger, H. Sevincli, H. Mehrez, and S. Ciraci, Phys. Rev. B 74, 235413 (2006).

${ }^{25}$ E. Lieb and D. Mattis, J. Math. Phys. 3, 749 (1962). 